\title{
Excimer Laser Ablation and Morphology of Uniaxially Stretched Poly(ethylene-2,6-naphthalate) Films
}

\author{
Tsunehisa Kimura, ${ }^{\dagger}$ Hisatsugu ToKunaga, Eiko Ito, \\ Hiroyuki NIINO,* and Akira YABE* \\ Department of Applied Chemistry, Tokyo Metropolitan University, \\ Minami-ohsawa, Hachioji, Tokyo 192-0397, Japan \\ * National Institute of Materials and Chemical Research, \\ Higashi, Tsukuba, Ibaraki 305-8565, Japan
}

(Received November 13, 1998)

\begin{abstract}
Microstructures formed by excimer laser ablation of uniaxially stretched poly(ethylene-2,6-naphthalate) (PEN) films were analyzed as a function of draw ratio, laser fluency, and number of pulses. Before ablation, stretched films with draw ratio higher than 3 exhibit band like structure on the surface running perpendicular to the drawing direction. By TEM observation of the cross section, this structure was found to extend into the bulk region. Upon laser pulse irradiation, microstructures are formed on the surface whose pattern and pitch are similar to those for the original band like structure. With further irradiation of pulses, pleated structures were formed with pitch larger than that for the original band like structure. Spectroscopic analysis of the pleated structure was conducted on the particles scratched off from the ablated surface. It was indicated that the pleated parts were amorphous but partially elongated. KEY WORDS Poly(ethylene-2,6-naphthalate)/Excimer Laser Ablation / Cold Drawing/Morphology/
\end{abstract}

Ablative photodecomposition (APD) occurs on irradiation of a pulsed excimer laser onto the surface of polymers, resulting in the formation of surface microstructures. $^{1-10}$ APD has drawn attention as a novel means for surface modification of polymeric films and fibers. Microstructures formed by APD depend on several factors including the power and wavelength of the laser, the number of pulses, type of polymers and manner of stretching the polymers. The effect of the stretching manner on the microstructures of crystalline polymers is of special interest in relation to the bulk morphology formed by stretching. Stretched crystalline polymers including poly(ethylene terephthalate) (PET) and poly(ethylene-2,6-naphthalate) (PEN) exhibit microstructures of the order of micrometers on APD, and the patterns of these microstructures definitely depend on whether the stretching is of uniaxial or biaxial. Unstretched amorphous films of these polymers produce a smooth surface. APD could thus probe the surface morphology to provide information about bulk morpho$\log$.

A number of studies have been reported on the structures of stretched PEN and PET. ${ }^{1-15} \mathrm{~A}$ point of discussion of these papers is the mesophase, ${ }^{16}$ which is expressed as a region rich in trans conformations but not crystallized. X-Ray diffraction and infrared spectroscopy suggest the existence of the mesophase, but spatial arrangement of this phase is not clear yet. APD sheds light on the morphology of the stretched crystalline polymers because (i) the etch rate by APD differs depending on the phase, and hence the resulting surface could reflect the morphology of the original surface, and (ii) especially when multiple pulses are applied, APD accompanies the melt and solidification affected by the bulk morphology.

This work reports the morphology of uniaxially

${ }^{\dagger}$ To whom correspondence should be addressed. stretched PEN films studied by APD. The morphology originally in the stretched films is studied in comparison to the pleated pattern formed by the ablation. The pleated parts are scratched off from the surface and a direct analysis on these scratched particles was carried out.

\section{EXPERIMENTAL}

\section{Sample Preparation}

Pellets of poly(ethylene-2,6-naphthalate) (PEN) $\left(M_{n}=\right.$ 17000) supplied by Teijin Co., Ltd. were dried and hot pressed at $300^{\circ} \mathrm{C}$ to obtain amorphous films ( $\mathrm{ca} .100 \mu \mathrm{m}$ in thickness). The amorphous films were cut into $10 \mathrm{~mm}$ by $50 \mathrm{~mm}$ strips. A strip was marked with scale marks (division of $1 \mathrm{~mm}$ ) on both edges, and stretched at $90^{\circ} \mathrm{C}$ in a water bath. The draw ratio $(D R)$ was determined by the ratio of final to initial divisions. For the unstretched strip, $D R=1$. Elongation proceeded with neck formation. Maximum attainable $D R$ was $c a .5$.

\section{Laser Ablation}

A $\mathrm{KrF}(248 \mathrm{~nm}$ ) laser (Lambda Physic) was used to irradiate the samples. The full width at half-maximum was approximately $30 \mathrm{~ns}$. The fluence was between 50 and $500 \mathrm{~mJ} \mathrm{~cm}{ }^{-2}$ pulse $\mathrm{m}^{-1}$ and the area of $c a .5 \mathrm{~mm}$ by $10 \mathrm{~mm}$ was irradiated. The frequency of the shots was $2 \mathrm{~Hz}$ unless otherwise mentioned.

\section{Fourier Transform Infrared Spectroscopy (FT-IR)}

FT-IR spectroscopy was conducted on a Nicolet Magna 750, with resolution of $2 \mathrm{~cm}^{-1}$, and 100 scans were averaged.

\section{$X$-Ray Measurement}

Wide angle X-ray diffraction measurements were carried out by an MAC Science MXP operated at $40 \mathrm{kV}$ and $300 \mathrm{~mA}$ to generate Ni-filtered $\mathrm{Cu}-K_{\alpha} \mathrm{X}$-ray beam. 


\section{Morphology Observation}

Morphology observation was carried out by a scanning electron microscope (SEM) (an Akashi Alpha-25A) and a transmitting electron microscope (TEM) (JEOL 2000FX).

\section{Differential Scanning Calorimetry (DSC)}

DSC was carried out using a SEIKO DSC200 equipped with a thermal analysis system SSC5200H under a dry nitrogen atmosphere. The heating rate was $10^{\circ} \mathrm{C} \mathrm{min}^{-1}$.

\section{Density Measurement}

Sample density was measured with a density gradient column prepared with a mixture of carbon tetrachloride and $n$-heptane.

\section{Chemical Etching}

Chemical etching was carried out as follows: $2.44 \mathrm{~g}$ orthophosphoric acid was dissolved in $20 \mathrm{ml}$ sulfuric acid, followed by the addition of $0.35 \mathrm{~g}$ potassium permanganate and mixed under sonification for $1 \mathrm{~h}$ to obtain an etching solution. The film sample was etched in this solution for $1 \mathrm{~h}$, followed by washing with dilute sulfuric acid solution, hydrogen peroxide solution, distilled water, and acetone, and drying overnight.

\section{RESULTS AND DISCUSSION}

\section{Effects of Bulk Structures}

The microstructures formed on the surface of the uniaxially stretched PEN films on laser irradiation are entirely different from those formed on the surface of the two-dimensionally stretched films. ${ }^{7}$ This suggests that there are some structures originally existing in the bulk or on the surface of the base film, which cause characteristic microstructures in the subsequent ablation process.

Figure 1a shows an SEM photograph taken for the surface of the original PEN film uniaxially stretched by 5 times (draw ratio, $D R=5$ ). The stretched film used here was almost amorphous as evident by the X-ray measurement. The photograph exhibits a band like structure running perpendicular to the stretching direction. No such structure was observed for films with $D R$ less than 3. Films with $D R<3$ are transparent, while those with $D R>3$ look slightly turbid. The TEM photograph (Figure 1b) of the cross section of the same film indicates that the band like structure is not restricted to the surface alone, but extends into the bulk region. TEM observation suggests that the band structure is of the alternation of two different regions with different densities. The alternating black and white regions observed in the photograph indicate the existence of the dense and less dense regions, each corresponding to the elongated and less elongated regions, respectively. A similar band like structure is reported ${ }^{17}$ for uniaxially stretched PEN though the pitch (several tens of nanometers) reported was smaller than our observation. The surface structure observed in Figure 1a is enhanced by a chemical etching of the surface as shown in Figure 1c. The pitch for the etched surface seems comparable to that in the cross section and on the non-etched surface.

Since the bulk structure is reflected on the surface (a)

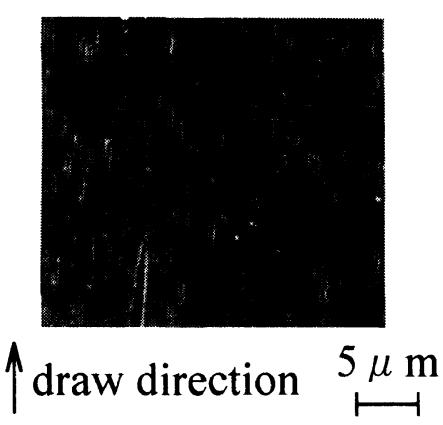

(b)

(c)
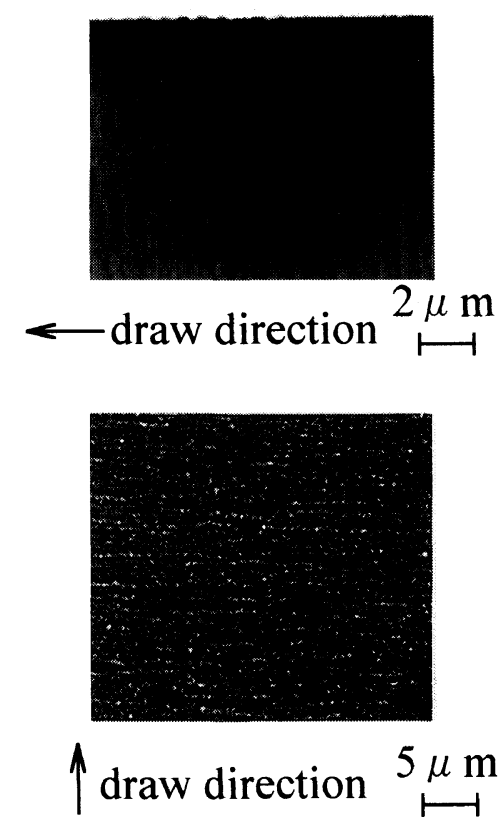

Figure 1. Surface morphology of uniaxially stretched PEN film $(D R=5)$ observed before ablation. (a) intact surface observed by SEM; (b) TEM photograph of the cross section of film cut parallel to the draw direction indicated in (a). The top corresponds to the surface observed in (a); (c) surface observed by SEM after chemical etching.

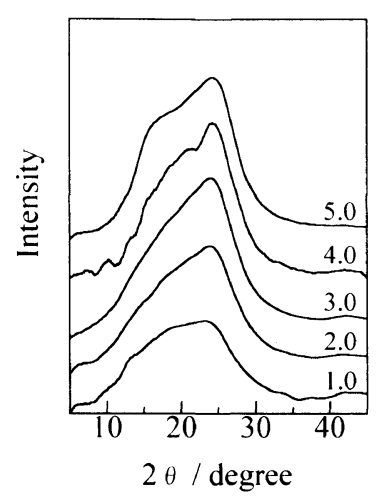

Figure 2. X-Ray equatorial scans (through view) measured for uniaxially stretched PEN films with various draw ratios.

structure as seen in Figure 1, the structure analysis of bulk should provide information on the surface. Figure 2 shows the X-ray diffraction profiles measured for PEN films with various draw ratios. The profiles indicate that these films are almost amorphous.

The infrared spectrum ${ }^{18,19}$ of the $\alpha$-form crystal of PEN exhibits bands at 2908, 2971, and $2990 \mathrm{~cm}^{-1}$. However, the spectra shown in Figure 3 do not exhibit the band at $2990 \mathrm{~cm}^{-1}$. With increase in the draw ratio, 


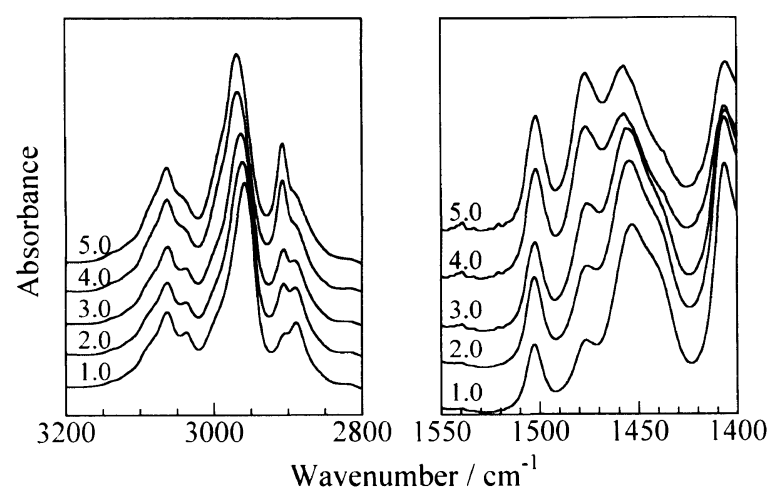

Figure 3. Infrared spectra obtained for uniaxially stretched PEN films with various draw ratios.

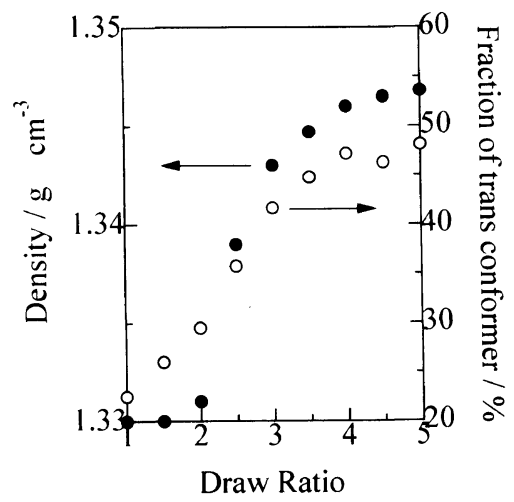

Figure 4. The fraction of trans conformer and density as a function of draw ratio.

the intensity of the band at $2908 \mathrm{~cm}^{-1}$ increases, but little increase is observed around $2990 \mathrm{~cm}^{-1}$ even for the films with higher draw ratios. The main peak around 2959 $\mathrm{cm}^{-1}$ observed for the non-stretched amorphous film shifts to a higher wave number upon stretching. In this range of wave number, the peak profiles for the films with larger draw ratios are more similar to the profile of the unstretched amorphous film $(D R=1)$ than to the profile of the $\alpha$-form crystal reported previously. ${ }^{19}$ These observations indicate that little crystallite is formed in the drawn films but may exist in small amount. Slight differences in the spectrum profiles might be attributed to the conformation change due to stretching. The bands at 1450 and $1477 \mathrm{~cm}^{-1}$ are assigned to the $\mathrm{CH}_{2}$ scissoring vibration of the gauche and trans conformations, respectively. ${ }^{18}$ Increase in trans conformation with the increase in draw ratio is obvious. This trans conformation is attributed to the elongated chains in the amorphous phase since little crystallite is assumed in the stretched films.

The fraction of the trans conformation is defined as the ratio of the peak height at $1477 \mathrm{~cm}^{-1}$ (trans) to the total of the heights at $1477 \mathrm{~cm}^{-1}$ and $1450 \mathrm{~cm}^{-1}$ (gauche). The trans fraction, together with the density, is shown in Figure 4. These properties increase with the draw ratio $(D R)$ and level off around $D R=4$. Under stretching in this study, a draw ratio of 5 was the maximum attainable elongation beyond which a fracture of the film occurred.

Figure 5 shows the DSC thermograms. The unstretched film $(D R=1)$ and the stretched film $(D R=2.0)$ exhibit exothermic peaks at 200 and $170^{\circ} \mathrm{C}$, respectively,

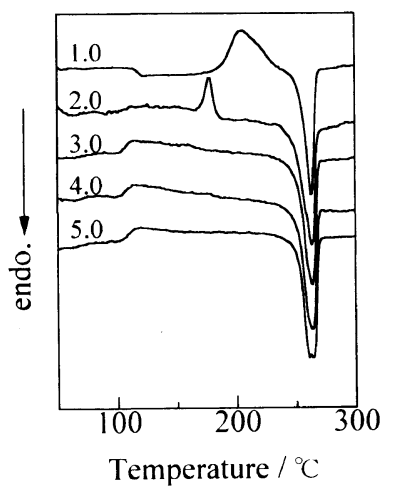

Figure 5. DSC thermograms for the uniaxially stretched PEN films with various draw ratios. Heating rate, $10^{\circ} \mathrm{C} \min ^{-1}$.

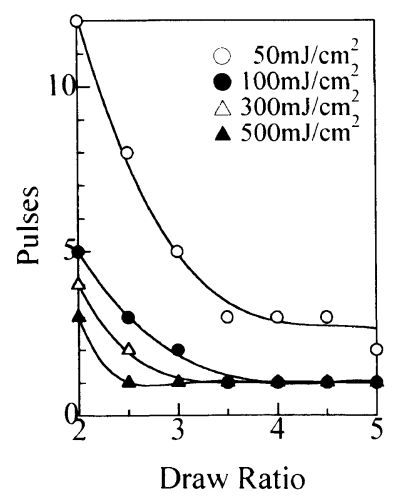

Figure 6. Minimum number of pulses required for pattern formation plotted against the draw ratio. Each symbol indicates the fluence of the laser per pulse.

due to the cold crystallization. For films with higher draw ratios, the exothermic peak overlaps with the change at $110^{\circ} \mathrm{C}$ associated with the glass transition and cannot be resolved clearly. In the unstretched film, polymer chains require a longer time to achieve the disentanglement necessary for subsequent formation of the crystalline phase. As a result, a relatively higher temperature is necessary for the cold crystallization to occur. In the stretched film, polymer chains are elongated resulting in the parallel alignment and reduction of chain entanglement. The polymer chains are not crystallized but readily crystallized if the chain mobility is enhanced by heating. This may be the case for the film with $D R=2$ whose cold crystallization temperature is lower than that for the unstretched film.

\section{Onset of Pattern Formation}

With laser irradiation, pleated structures running perpendicular to the stretching direction show up on the surface. The onset condition depends on the fluence of the laser, the number of pulses, and the draw ratio of the film. In Figure 6, the minimum number of pulses required for the onset of the pattern formation is plotted against the draw ratio. There are three distinguishable regions: (i) the lowest fluence $\left(50 \mathrm{~mJ} \mathrm{~cm}{ }^{-2}\right.$ pulse $\left.^{-1}\right)$ for which more than one pulse is required for the pattern formation, (ii) higher fluence values $\left(100\right.$ to $500 \mathrm{~mJ} \mathrm{~cm}^{-2}$ pulse $\left.^{-1}\right)$ with higher draw ratios $(D R=3$ to 5$)$ for which one pulse is sufficient for pattern formation, and (iii) higher fluence values ( 100 to $500 \mathrm{~mJ} \mathrm{~cm}^{-2}$ pulse $^{-1}$ ) and lower draw ratios $(D R=2$ to 3$)$. The $50 \mathrm{~mJ} \mathrm{~cm}^{-2}$ 
(a)

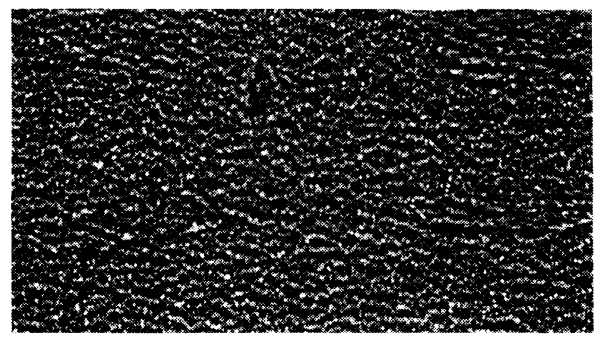

\section{$\uparrow$ draw direction $\stackrel{5 \mu \mathrm{m}}{\longmapsto}$}

(b)

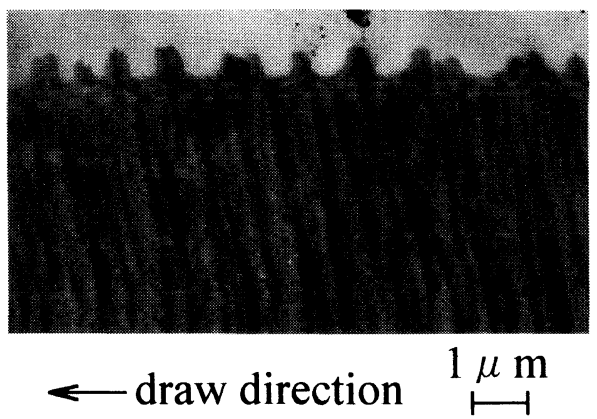

Figure 7. Microstructure formed on the film surface $(D R=5)$ at the onset condition $\left(500 \mathrm{~mJ} \mathrm{~cm}^{-2}\right.$ pulse $\mathrm{c}^{-1}, 1$ pulse) of laser irradiation: (a) surface observed by SEM and (b) cross section observed by TEM.

pulse $^{-1}$ is close to the reported threshold value ${ }^{8}$ below which little ablation occurs with one pulse. $D R=3$ is the limiting value above which a band like structure is observed in the original uniaxially stretched films.

SEM and TEM photographs taken after the laser irradiation are shown in Figure 7. The irradiation condition is the lowest limit necessary for pattern formation ( $500 \mathrm{~mJ} \mathrm{~cm}{ }^{-2}$ pulse $^{-1}, 1$ pulse, corresponding to the category (ii)). The pitch of the pleated pattern is about $1-2 \mu \mathrm{m}$, slightly larger but close to the pitch of the band like structure observed on the chemically etched surface of the original uniaxially stretched film. This may indicate that the etching by ablation is the main process at this condition of the laser irradiation. Selective elimination of one of the two regions in the original band like structure occurs, resulting in a surface microstructure. However, the pattern formed is not exactly the same as that by chemical etching and the pitch is slightly larger, suggesting that melting and solidification are involved in ablation. At present, it is difficult to know whether elongated or amorphous regions is etched more to form the ablated pattern, but a study on $\mathrm{PET}^{6}$ seems to indicate that the amorphous region is eliminated selectively.

It should be noted that the band like structure is not observed in the original stretched films with $D R$ smaller than 3. Nevertheless, the pleated structure occurs with laser irradiation (category (iii) and category (i) with small $D R \mathrm{~s}$ in relation to Figure 6). The reason for this is not clear at present, but a possible explanation is as follows: Though the band like structure is not clearly observed for the films with small $D R \mathrm{~s}$, the elongation of the chain is evident because increase in the trans fraction is observed as shown in Figure 4. Pulses impinged prior to the onset of the pattern formation may cause some (a)

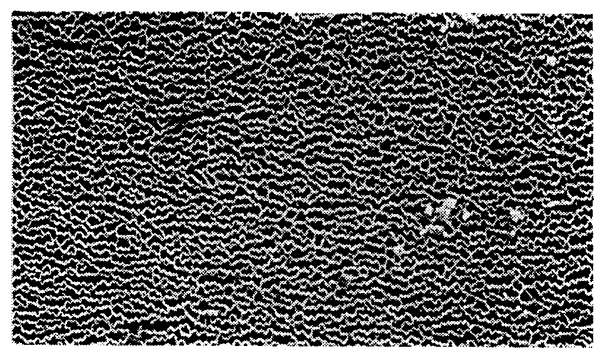

(b)

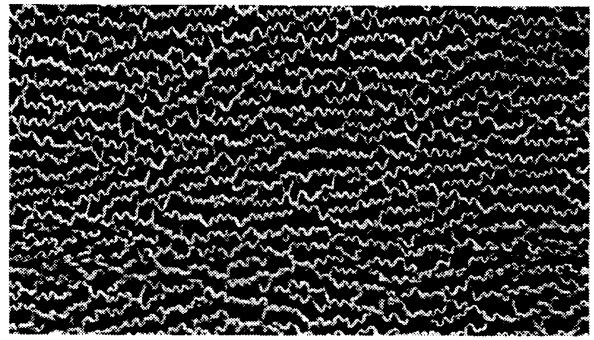

(c)

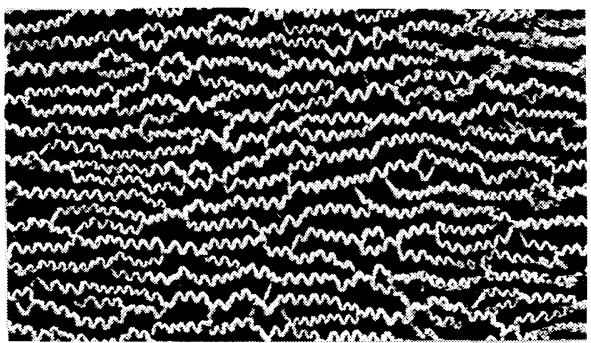

$$
\uparrow \text { draw direction } \stackrel{20 \mu \mathrm{m}}{\longmapsto}
$$

Figure 8. Evolution of surface morphology, observed with SEM, of the uniaxially stretched PEN film with $D R=5$ upon increase in number of pulses: (a) 10 pulses, (b) 20 pulses, and (c) 100 pulses. Fluence is $500 \mathrm{~mJ} \mathrm{~cm}^{-2}$ pulse $^{-1}$.

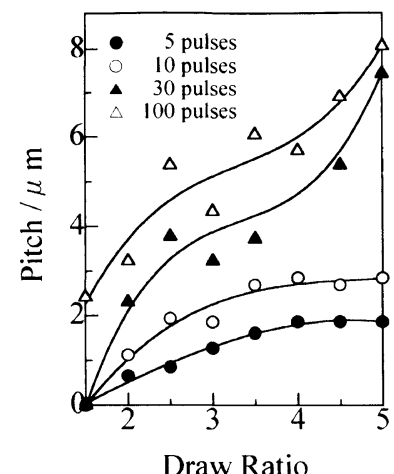

Figure 9. Pitch of pleated structures as a function of draw ratio. Fluence is $500 \mathrm{~mJ} \mathrm{~cm}^{-2}$ pulse $^{-1}$.

structure change on the surface, possibly in a way to enhance inhomogeneity, encouraging the formation of the microstructure. This is a speculation and should be examined in the future study.

\section{Evolution of Patterns}

The pitch of the pleated pattern increases with increase in the pulse number as shown in Figure 8 . The increase in pitch is clearly observed. It is shown in Figure 9 that the pitch obtained after the irradiation of multiple pulses depends on the $D R$. For smaller pulse numbers ( 5 and $10), D R$ dependence of pitch is similar to that of the 
(a)

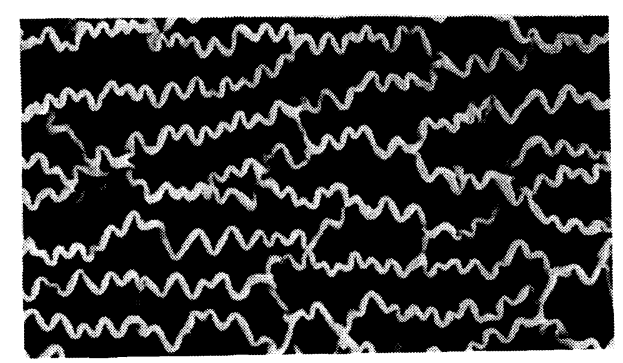

(b)

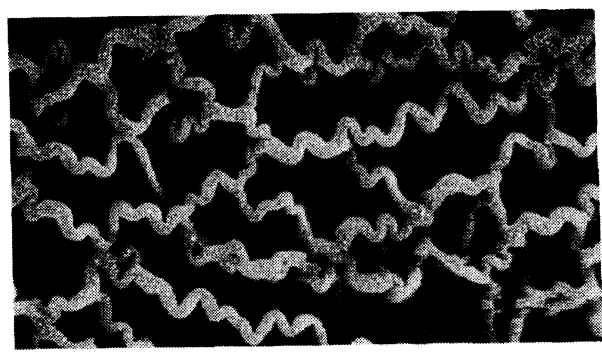

\section{$\uparrow$ draw direction $\stackrel{10 \mu \mathrm{m}}{\longmapsto}$}

Figure 10. Frequency dependence of the surface morphology: SEM photographs obtained for (a) $1 \mathrm{~Hz}$ and (b) $10 \mathrm{~Hz}$, with $500 \mathrm{~mJ} \mathrm{~cm}^{-2}$ pulse ${ }^{-1}, 50$ pulses.

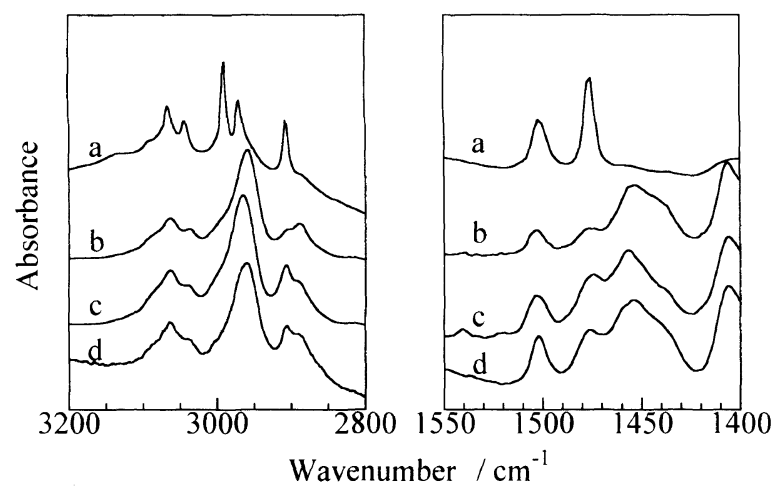

Figure 11. Infrared spectra of (a) crystalline phase, (b) amorphous film, (c) uniaxially stretched film $(D R=2.5)$, and (d) particles scratched off from the pleated surface formed by the ablation at $500 \mathrm{~mJ} \mathrm{~cm}^{-2}$ pulse $^{-1}$ with 100 pulses.

trans fraction and the density as was shown in Figure 4, indicating that the base film structure affects the pattern. In the case of more pulse numbers (30 and 100), $D R$ dependence is slightly different. The frequency of the pulse also affects the pitch. As show in Figure 10, high frequency causes a larger pitch.

The pitch observed for the films with multiple irradiation or a higher frequency is evidently larger than the pitch observed at the onset of the pattern formation $(1-2 \mu \mathrm{m})$. This indicates that selective etching by ablation is no longer the main process of pattern evolution. It is most likely that the melt and solidification play important roles during the evolution of patterns. Although the base film structure affects the pleated structure formed during the evolution, it seems that there is no direct relation between the pitch of the pleated

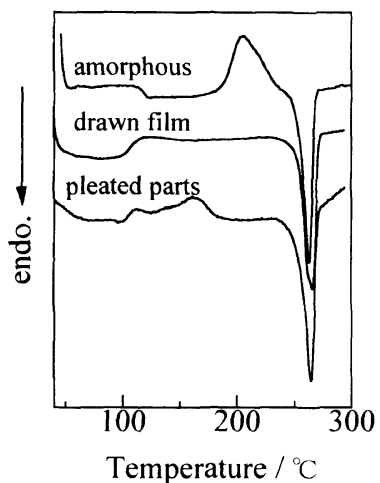

Figure 12. DSC thermograms for scratched particles, along with those for amorphous film and uniaxially stretched film $(D R=5)$.

pattern and that of the band like pattern existing in the original stretched film.

\section{Analyses of the Pleated Structure}

To analyze the pleated structure directly, the surface of the film after the laser irradiation $\left(D R=5500 \mathrm{~mJ} \mathrm{~cm}^{-2}\right.$ pulse $^{-1}, 100$ pulses) was scratched with a razor blade and the pleated parts were collected. The scratched particles were subjected to the infrared, X-ray, and thermal analyses.

X-ray analysis shows that the pleated parts are amorphous. Figure 11 shows the infrared spectrum of the scratched particles, as well as the spectra for the crystalline phase, the uniaxially stretched film, and the amorphous phase. Comparison of the spectrum profiles clearly indicates that the pleated part is partially elongated but not crystallized. This observation is supported by DSC analysis of the scratched particles as is shown in Figure 12. Appearance of the cold crystallization peak around $160^{\circ} \mathrm{C}$ indicates a lower degree of orientation in comparison to the $D R=5$ of the original uniaxially stretched film.

The pleated structure, therefore, is not related directly to the elongated region in the original stretched film, nor does it seem plausible to relate the amorphous region in the original film to the pleated structure. It is most likely that the melting and the solidification occur during multiple pulse irradiation, causing the solidified pattern affected by the base film morphology.

\section{CONCLUSIONS}

(i) A band like structure was observed by SEM measurements on the surface of the uniaxially stretched PEN films with the draw ratio $(D R)$ larger than 3 . This structure was observed more clearly by chemical etching of the surface. TEM observation of the cross section indicated that the band like structure extends to the bulk. The alternating black and white regions observed in a TEM photograph indicated the existence of dense and less dense regions, each corresponding to the elongated and less elongated regions, respectively.

(ii) The minimum number of pulses necessary for the onset of the surface pattern formation depended on the fluence of the laser and the draw ratio of the base film. The pitch and the shape of the pleated structures formed on the surface under the onset condition was similar to 
the surface pattern observed prior to the ablation. This indicates that the etching by APD is the main process in the initial stage of pattern formation although melting and solidification are involved.

(iii) With increase in pulse number, the microstructure grew, resulting in increase in pitch and the regularity. Since the pitch of the pleated structure formed after the irradiation of a number of pulses was far larger than the originally existing one, there is no direct relation between the pleated structure and the band like structure. However, change of the periodicity was strongly dependent on the draw ratio of the films. This implies that the bulk morphology affects the formation process of the surface pattern during repeated melting and solidification occurring in association with ablation.

Acknowledgments. The authors thank to Teijin Co., Ltd. for supplying PEN samples.

\section{REFERENCES}

1. R. Srinivasan and V. Mayne-Banton, Appl. Phys. Lett., 41, 576 (1982).

2. R. Srinivasan and B. Braren, Chem. Rev., 89, 1303 (1989).

3. S. Lazare and V. Granier, Laser Chem., 10, 25 (1989).
4. S. Lazare and R. Srinivasan, J. Phys. Chem., 90, 2124 (1986).

5. T. Bahners and E. Schollmeyer, Angew. Makromol. Chem., 151, 19 (1987).

6. Y. Novis, J. J. Pireaux, A. Brezini, E. Petit, R. Caudano, P. Lutgen, G. Feyder, and S. Lazare, J. Appl. Phys., 64, 365 (1988).

7. H. Niino, M. Nakano, S. Nagano, A. Yabe, H. Moriya, and T. Miki, J. Photopolym. Sci. Technol., 2, 133 (1989).

8. H. Niino, A. Yabe, S. Nagano, and T. Miki, Appl. Phys. Lett., 54, 2159 (1989).

9. H. Niino and A. Yabe, J. Photochem. Photobiol. A: Chem., 65, 303 (1992).

10. H. Watanabe, T. Takata, and M. Tsuge, Polym. Int., 31, 247 (1993).

11. R. Jakeways, J. L. Klein, and I. M. Ward, Polymer, 37, 3761 (1996).

12. A. Ajji, J. Guevremont, K. C. Cole, and M. M. Dumoulin, Polymer, 37, 3707 (1996).

13. S. Murakami, M. Yamakawa, M. Tsuji, and S. Kohjiya, Polymer, 37, 3945 (1996).

14. M. Cakmak and S. W. Lee, Polymer, 36, 4039 (1995).

15. S. R. Padibjo and I. M. Ward, Polymer, 24, 1103 (1983).

16. B. Wunderlich, M. Moller, J. Grebowicz, and H. Baur, Adv. Polym. Sci., 87, 1 (1988).

17. A. Hirata, T. Fuchigami, T. Komoto, S. Mori, and K. Kitao, Polym. Prepr., Jpn., 42, 1406 (1993).

18. I. Ouchi, M. Hosoi, and S. Shimotsuma, J. Appl. Polym. Sci., 21, 3445 (1977)

19. F. Kimura, T. Kimura, A. Sugisaki, M. Komatsu, H. Sata, and E. Ito, J. Polym. Sci., Part B, Polym. Phys., 35, 2741 (1997). 